\title{
Ultra-high energy cosmic ray detector TUS: preliminary results of the first year of measurements
}

\author{
Pavel Klimov* \\ Lomonosov Moscow State University, Skobeltsyn Institute of Nuclear Physics (SINP MSU), \\ Moscow, Russia \\ E-mail: pavel.klimov@gmail.com
}

For the Lomonosov-UHECR/TLE Collaboration

TUS (Tracking Ultraviolet Set-up) is the first orbital detector of extreme energy cosmic rays. It was launched into orbit on April 28, 2016, as a part of the scientific payload of the Lomonosov satellite. The main aim of the mission is to test the technique of measuring UV fluorescent and Cherenkov radiation of extensive air showers (EAS) generated by primary cosmic rays with energies above $50 \mathrm{EeV}$. The first year of operation has revealed an unexpectedly rich diversity of UV radiation. Among different types of registered events, we have identified those caused by thunderstorm activity and transient luminous events (TLEs), anthropogenic factors and by lowenergy cosmic rays passing through the UV glass filters covering the focal plane. Three modes of operation aimed at studying TLEs and meteors were also tested. A search and analyses of EAS candidates is in progress. We report the preliminary results obtained during the first year of TUS operating in orbit in all modes of operation with a special attention to the EAS mode.

35th International Cosmic Ray Conference - ICRC2017

10-20 July, 2017

Bexco, Busan, Korea

\footnotetext{
*Speaker.
} 


\section{Introduction}

Measurements of the cosmic ray (CR) energy spectrum, their nuclear composition and arrival directions at extreme energies $E \gtrsim 50 \mathrm{EeV}^{1}$ are an important part of modern astrophysics and particle physics [1].

The first CR particles of such great energy were detected [2] and a cut-off of the energy spectrum was predicted $[3,4]$ more than 50 years ago but results in the EECR study do not give clear answers to the most important question of the Greisen-Zatsepin-Kuzmin cut-off [5]. The nature and origin of EECRs are still not understood. To a great extent, the problem relates to a very low flux of EECRs: the two largest ground-based arrays-The Pierre Auger Observatory and the Telescope Array-registered less than two dozen events with energies $E>100 \mathrm{EeV}$ in 10 and 5 years of operation respectively $[6,7]$.

A primary goal of the TUS project, first announced in 2001 [8], is to expand the EECR experimental studies to space as was suggested by Benson and Linsley in early 1980's [9, 10]. The main idea is that fluorescent and Cherenkov ultraviolet (UV) radiation of an extensive air shower (EAS) generated by an EECR in the nocturnal atmosphere of the Earth can be detected from a satellite similar to the way it is observed from the ground but with a much larger exposure, thus considerably increasing the statistics of registered events.

Following the idea, a number of orbital detectors with a large signal collecting area and high time-lateral resolution are being elaborated [11]. The TUS detector is a pathfinder to the large-scale missions like KLYPVE [12] or JEM-EUSO [13]. Accurate estimations of the energy and arrival directions of EECRs are left to the future orbital detectors.

Skobeltsyn Institute of Nuclear Physics of Lomonosov Moscow State University (SINP MSU), Joint Institute for Nuclear Research (JINR) and Space Regatta Consortium together with several Korean and Mexican Universities have collaborated in the TUS detector preparation. Results of the detector simulations, development and preflight tests are published elsewhere [14, 15, 16, 17, 18].

The TUS detector on board Lomonosov satellite was launched into orbit from the newly built Vostochny Cosmodrome (Russia) on April 28, 2016. The satellite has a sun-synchronous orbit with an inclination of $97^{\circ} .3$, a period of $\approx 94 \mathrm{~min}$, and a height of about $470-500 \mathrm{~km}$.

\section{The TUS detector data acquisition system and on-flight trigger}

The TUS detector on board Lomonosov satellite is presented in Fig. 1. It consists of two main parts: a modular Fresnel mirror-concentrator and a photo-receiver matrix composed of 256 channels (16 photodetector modules, PDMs, of 16 channels each) in the focal plane of the mirror. A sensor of each channel (pixel) is a Hamamatsu R1463 photomultiplier tube (PMT) with a $13 \mathrm{~mm}$ diameter multi-alcali cathode covered by an UV glass filter and a reflective light guide with a square entrance of $15 \mathrm{~mm}$ size. Quantum efficiency of PMTs in the near UV (300-400 nm) band is $\sim 20 \%$. The Fresnel mirror has an area of $\sim 2 \mathrm{~m}^{2}$ and a focal distance of $1.5 \mathrm{~m}$. One pixel has a field of view (FOV) of $10 \mathrm{mrad}$, which corresponds to a spatial spot of about $5 \mathrm{~km} \times 5 \mathrm{~km}$ at the sea level for a $500 \mathrm{~km}$ orbit height. Thus, the total FOV equals $\pm 4.5^{\circ}$, and the full area observed by TUS at

\footnotetext{
${ }^{1} 1 \mathrm{EeV}=10^{18} \mathrm{eV}$. We will call particles with energies more than $50 \mathrm{EeV}$ extreme energy cosmic rays (EECRs).
} 
any moment is approximately $80 \mathrm{~km} \times 80 \mathrm{~km}$. A detailed description of the TUS detector can be found in [19].

The general design of TUS is determined by its main task of registering fluorescence and scattered (reflected) Cherenkov radiation of EASs in the near UV band with a time resolution of $0.8 \mu \mathrm{s}$ in full temporal interval of 256 time steps.
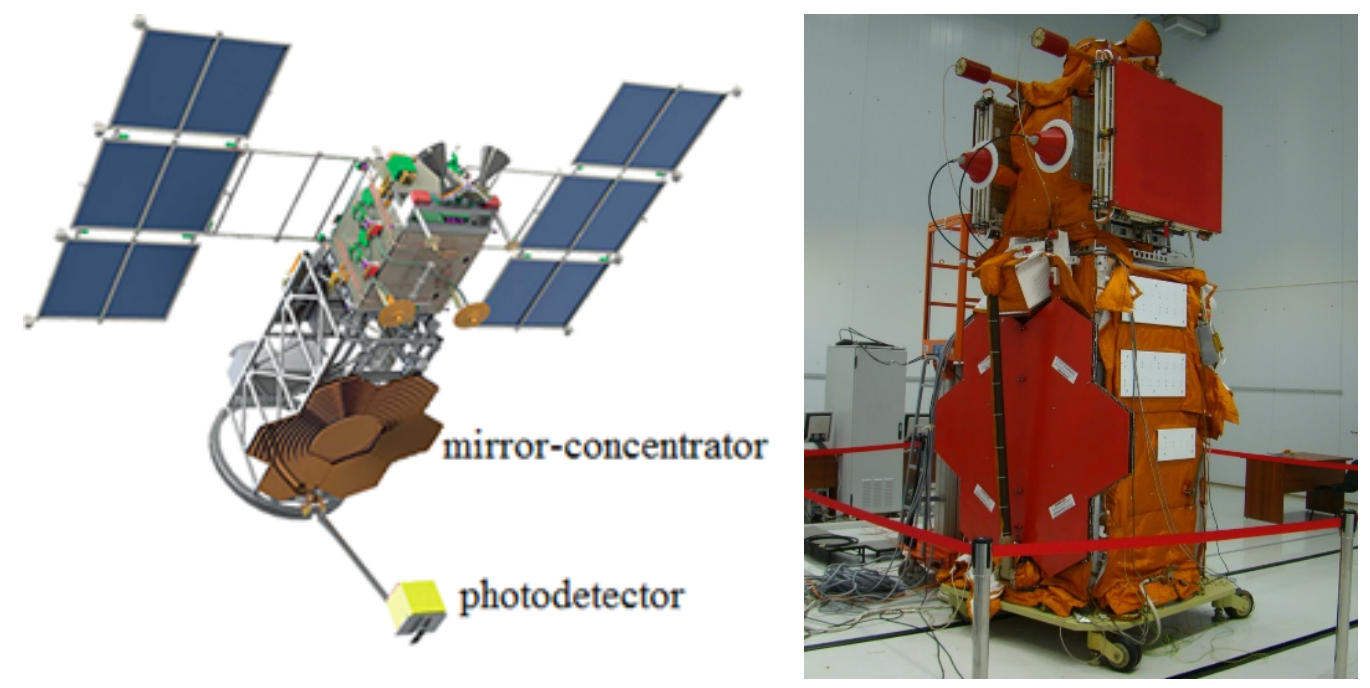

Figure 1: Left: artist's view of the TUS detector on-board the Lomonosov satellite. Right: the TUS detector assembled on the Lomonosov frame before the launch.

TUS has a two-level trigger $[20,21]$. The first-level trigger is a threshold one: the PDM board calculates a moving sum (MS) of PMT signals during 16 consecutive time steps in each channel and looks for an MS value above a threshold level (active pixel). The threshold level is calculated as $\langle M S\rangle+d Q$, where $\langle M S\rangle$ is the mean value of MS obtained during previous $100 \mathrm{~ms}$ for each channel individually, and $d Q$ is a predefined constant (unique for all channels) which can be changed by commands from the mission control center (MCC). The PDM board transmits a trigger information to the central processor board (CPB), which forms a map of active pixels. The second-level trigger is a pixel-mapping one implemented in the CPB. It acts as a contiguity trigger. This procedure selects cases of sequential triggering of spatially adjacent active pixels that are also contiguous in time, allowing for the selection of events with a specific spatial-temporal pattern. An additional parameter important for this trigger is the so-called adjacency length $(L)$, i.e., the number of neighboring channels sequentially activated by a signal. Data of all 256 channels for 256 consecutive time steps is transmitted from the CPB memory to the on-board computer in case conditions of the trigger system are satisfied.

For the maximal EAS trigger rate of about $1 \mathrm{~min}^{-1}$ (confined by the design of the on-board computer) and the event size of $128 \mathrm{KiB}$, an amount of data collected in one satellite revolution does not exceed $12 \mathrm{MiB}$. This information is transferred to the MCC twice per day.

It is important to note that the TUS electronics can operate in four modes intended for detecting various fast optical phenomena in the atmosphere at different time scales with different time sampling. Collected waveforms in each mode consist of 256 time samples. The main mode, as described above, is aimed at registering EASs born by EECRs and has a time sampling of $0.8 \mu \mathrm{s}$. 
This mode is also efficient for measurements of fast transient atmospheric phenomena - elves, the most common and fastest (less then $1 \mathrm{~ms}$ duration time) type of transient luminous events (TLEs). Next two modes have time sampling of $25.6 \mu \mathrm{s}$ and $0.4 \mathrm{~ms}$ and are suitable for studying other types of TLEs slower than elves: sprites, blue jets, gigantic jets, etc. The last mode has 6.6 ms sampling time and can detect (micro)meteors, space debris and thunderstorm activity at a longer time scale $(\sim 1.7 \mathrm{~s})$.

Digital integration of the signal and measurements at various time scales allow studying transient atmospheric phenomena of different origin and using the TUS detector as a multifunctional space observatory for astrophysical and geophysical research [22, 23, 24]. Examples of measurements in slow modes are presented in the last section of the article.

\section{EAS mode measurements}

TUS began measurements in space on May 19, 2016. The first months of work were mostly dedicated to testing hardware, software and various modes of operation. Continuous measurements were started in September 2016 with several gaps for the satellite technical service. By the end of May 2017, TUS registered around 35,000 events at nocturnal parts of its orbit in the EAS mode. The total exposure is approximately $530 \mathrm{~km}^{2} \cdot \mathrm{yr} \cdot \mathrm{sr}$. It is small in comparison with that of the largest ground-based arrays but sufficient to look for an EECR above the TUS threshold energy $E \gtrsim 70 \mathrm{EeV}[21]$.

Various aspects of the TUS operation in space were simulated before the launch [25, 20, 26, 21]. The simulations employed ESAF (EUSO Simulation and Analysis Framework) [27] and the TUSSIM program developed at JINR. The QGS model parametrization of Ilina et al. [28] was selected as the closest to experimental data at primary energies in the range of 10-100 EeV. These simulations allow to study the EAS signal temporal structure and expected amplitude and to develop the criteria for the EAS search in the TUS detector data. Temporal structure and amplitude of the signal are significantly different for "horizontal" EAS $\left(60^{\circ}<\theta<90^{\circ}\right)$ and "vertical" EAS $\left(0^{\circ}<\theta<30^{\circ}\right)$. For this reason the developed criteria for EAS search are different for these types of events.

\subsection{EAS signal simulation and estimations}

The duration of a horizontal EAS in the FOV of one pixel is $\sim 20 \mu$ s. For vertical EASs, it is approximately two times larger. The total duration of an EAS signal depends on the zenith angle and varies approximately from $30 \mu$ s to $100 \mu \mathrm{s}$. A measure of the energy of a primary particle is a number of photons $N_{\text {ph }}$ in the EAS maximum, which is related to the electron (positron) number $N_{\mathrm{e}}$ as $N_{\mathrm{ph}}=Y N_{\mathrm{e}} \cdot c \Delta t$, where $Y \sim 4.5$ photons $/ \mathrm{m}$ is a fluorescent photon yield (approximately constant at heights 3-12 km in the atmosphere), $c$ - speed of light, $\Delta t$ - time interval of measurements. For a horizontal EAS generated by a $100 \mathrm{EeV}$ proton the detected amplitude of fluorescence at the cascade maximum is expected to be of the order of 20 photons $/ \mu \mathrm{s}$.

It should be noticed that maximum of the vertical cascade curve at such high energies is close to the sea level, so that a considerable part of the cascade curve escapes observation in the atmosphere. On the other hand, it is followed by a strong Cherenkov light signal reflected by the surface (typical surface albedo of water is 5\%, sand and grass have an average albedo of $13 \%$ and $2 \%$ 
respectively while albedo of snow surface is up to 90\% [29]). A back-scattered Cherenkov "point" with an amplitude of the signal in one time sample of the detector much higher than the background is a characteristic mark of a vertical EAS.

Figure 2 demonstrate results of simulations of the flux of photons in the TUS detector channels for a vertical $\left(\theta=15^{\circ}\right)$ and horizontal $\left(\theta=75^{\circ}\right)$ air showers, zero background radiation is assumed. In both cases, the EASs are generated by a $100 \mathrm{EeV}$ primary proton arriving along one of the diagonals of the FOV. For the vertical EAS one can see features discussed above: the duration of the signal in the brightest pixel is $\approx 50 \mu$ s with a very short $(<1 \mu \mathrm{s}$ ) flash of the Cherenkov light at the end. This vertical EAS signal is expected to be measured in one or two pixels of an "ideal" detector with a small point spread function (PSF). In the real TUS detector, the PSF spreads for more than one pixel, and the signal might be registered in a group of 3-5 adjacent pixels.

A horizontal EAS shown in right panel of Fig. 2 demonstrates a completely different signal. In contrast to the vertical EAS, signals in separate channels have shorter duration with 20 photons $/ \mu \mathrm{s}$ in the brightest pixels. Information that can be obtained from inclined showers is richer than from vertical ones due to the signal detection in several pixels and larger signal-to-noise ratio due to shorter duration of useful time interval for EAS photons collection. An analysis of the light curves of different types of EASs allows one to obtain several criteria for selecting EECR candidates in the TUS data.
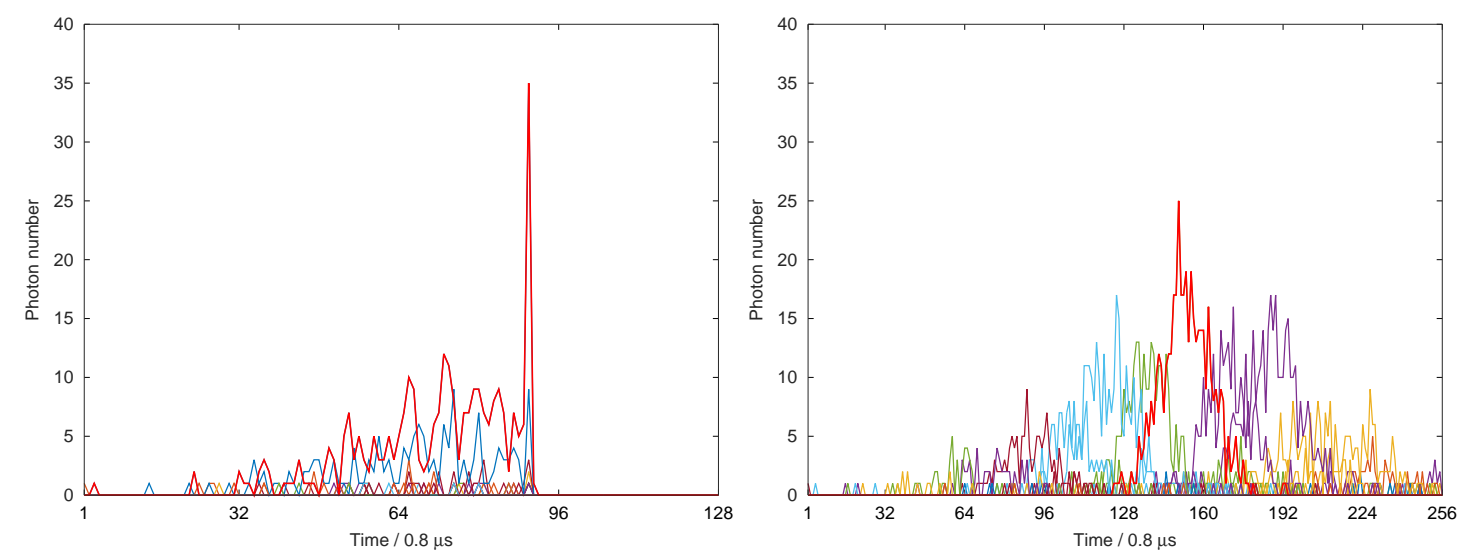

Figure 2: Photon flux on the TUS focal surface for a simulated EAS event from a $100 \mathrm{EeV}$ proton. Colors denote different channels, zero background is assumed. Left panel: vertical event, the Cherenkov peak at the end corresponds to the albedo of 0.05 . Right panel: horizontal event.

\subsection{EAS events criteria and candidates in the TUS data}

In a search for possible EAS candidates, we analyzed approximately 10,000 events registered in the conditions of the minimal background radiation and considered those that satisfied the following criteria. First, we selected events such that the moving sum of a waveform in the event exceeded the background level by at least 96 ADC counts ${ }^{2}$, and the adjacency length $L \geq 6$ (the same length was used in the on-flight level 2 trigger) and $L<17$ (to exclude comparatively slow non-EAS events).

\footnotetext{
${ }^{2} d Q=96$, i.e. signal in one time sample exceeds the base-level on 6 ADC counts in average.
} 
Next part of the constraints is based on the Gaussian approximation of waveforms of active pixels ("fit conditions"):

- the peak of the Gaussian fit was located within the record (excluding first 90 and last 30 time steps);

- total duration of the signal in any active pixel was within 30-180 time steps;

- the coefficient of multiple determination of a multiple linear regression $R^{2}>0.8$.

The above criteria were developed basing on simulations of a few thousand EECRs with different parameters of primary particles and an analysis of different types of "noise" events in the data set [24]. The procedure led us to a list of 13 events presented in [30].

The next step of the analysis is an event by event study of the temporal and spatial dynamics of the signal. This analysis is aimed to search for typical signatures of an EAS taking into account the characteristic temporal parameters, signal amplitudes and image structure discussed above. The latter one is mainly determined by the PSF, which is larger than a pixel size and differs in various parts of the FOV. The preflight measurements of the PSF showed that is has a diameter of 27$30 \mathrm{~mm}$ ( $70 \%$ of energy) on the edge of the FOV, which is approximately twice larger than the size of a pixel [31]. Simple estimations show that it can increase the signal duration in 1.5-2 times in comparison with an ideal optical system. For a more accurate determination of the temporal parameters of a signal (duration in a separate channel and the total duration of the light curve), their evaluation was made by means of the Full Duration at Half Maximum (FDHM).

As was shown above, it is expedient for the subsequent analysis to formulate two different sets of the second level criteria, separately for horizontal and vertical events.

Horizontal EAS criteria:

1. Track-like image: alignment of 5 or more active pixels in a line ("track").

2. The characteristic duration of the signal in one channel: $20-50 \mu \mathrm{s}$.

3. The characteristic total duration of the signal: more than $50 \mu \mathrm{s}$.

\section{Vertical EAS criteria:}

1. Spot-like image: the signal is confined to $2-4$ neighboring pixels.

2. The characteristic duration of the signal in one channel: more than $40 \mu \mathrm{s}$.

3. The characteristic total duration of the signal: less than $50 \mu \mathrm{s}$.

4. Presence of the Cherenkov peak at the end of the light curve during one time step and not later than in $10-30 \mu \mathrm{s}$ after the maximum of the EAS fluorescence.

As a result of this event by event analysis, a number of events were eliminated from the further consideration. Due to lower UV radiation intensity of vertical EAS their registration probability is significantly suppressed. The majority of EAS-like events can be related to fast anthropogenic signals (for example, airports). Here we present the most reliable and strong candidates for the horizontal EAS produced by an EECR particle. 
The first candidate is presented in Fig. 3 - event 20161003-054859 (this is event \#3 from the list of [30]). It has the most interesting spatial-temporal dynamics similar to what is expected for an EAS (to minimize statistical fluctuations we express signal as moving average with width parameter $M=16$ ). Active pixels are grouped in an oblong spot, the shape of which might be a "convolution" of two factors-the mirror PSF and a linear track.

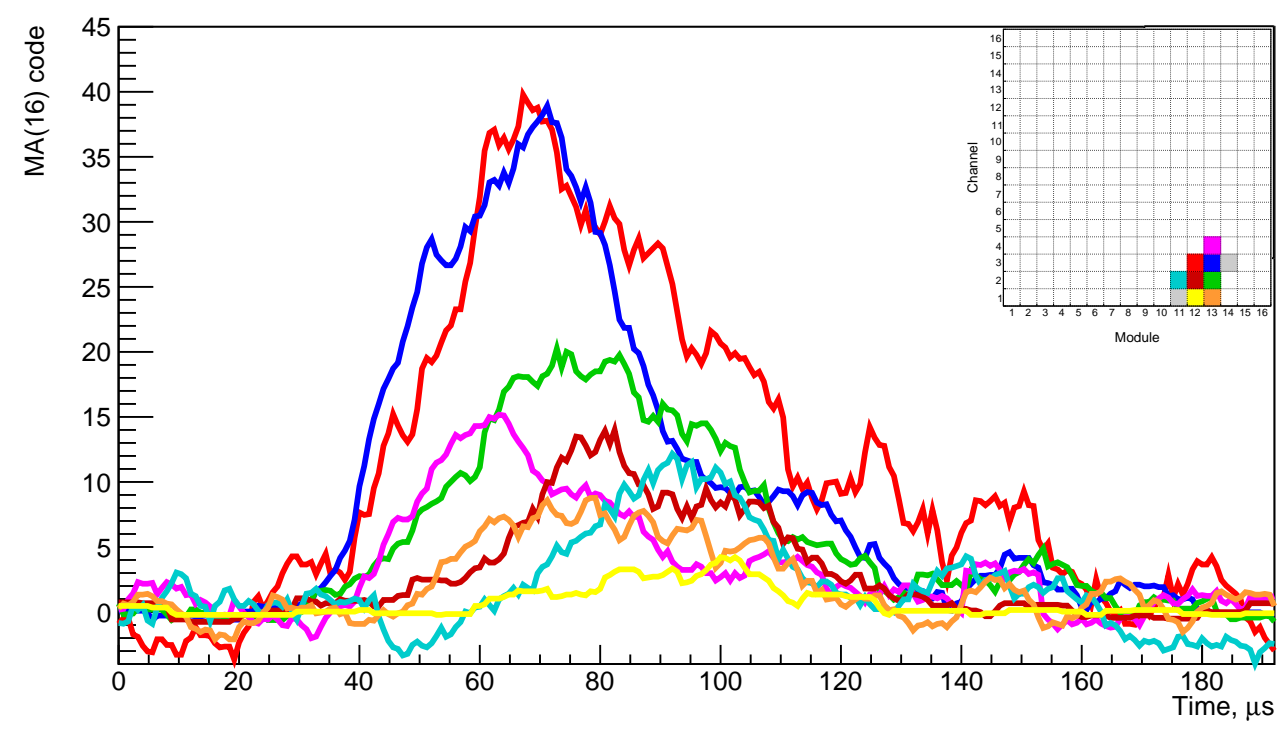

Figure 3: Event 20161003-054859: moving average of ADC counts for active pixels (individual baselevels are subtracted) and corresponding pixel map.

Main information about active pixels and temporal characteristics of the signal are listed in Table 1. It can be seen from the waveforms that characteristic duration of the signal is $70-100 \mu \mathrm{s}$ which is significantly larger than one can expect from a vertical EAS (but can be explained with PSF-effect elongation for horizontal event). The moment of the maximum of the signal in each pixel has some shift from one pixel to another which can be interpreted as EAS image movement. This is an argument in favour of a horizontal (inclined) EAS origin for this event.

It is important to mention that a possible thunderstorm activity was studied in the region of this event measurements. The Vaisala GLD360 ground based lightning location network [32, 33] did not register any lightning strikes in a region with radius of $930 \mathrm{~km}$ and during $10 \mathrm{~s}$ period around the time of the TUS event. This provides a strong support for a non-thunderstorm origin of the event.

Second very promising example of EAS candidates is event 20161031-102518 which TUS detected above Alaska $\left(61^{\circ} .31 \mathrm{~N}, 155^{\circ} .7 \mathrm{~W}\right)$. It has 9 active pixels with the same feature: characteristic shifts of the spatial-temporal development. We presented another variant of signal presentation on the Fig. 4 - individual signals (moving average with parameters $M=3$ shifted by baselevel in this case) of each active pixels are stacked in one histogram, so that its envelope represents full light curve of the event.

Quantitative estimates of the EAS parameters for these two events and other EAS candidates are under consideration and will be published elsewhere. 
Table 1: Parameters of the event 20161003-054859: location of the active pixels (module and channel numbers), background level $A_{\mathrm{bg}}$ and peak amplitude $A_{\max }$ (in ADC codes), time $T_{\max }$ of the peak from the beginning of the record, and the FDHM of the signal $T_{1 / 2}$. All parameters are estimated by the moving average for 16 time samples.

\begin{tabular}{|c|c|c|c|c|}
\hline $\mathrm{md} / \mathrm{ch}$ & $A_{\mathrm{bg}}$ & $A_{\max }$ & $T_{\max }, \mu \mathrm{s}$ & $T_{1 / 2}, \mu \mathrm{s}$ \\
\hline $13 / 4$ & 5.7 & 15 & 63 & 36 \\
$12 / 3$ & 7.2 & 40 & 67.5 & 47 \\
$13 / 3$ & 0.9 & 38 & 71.5 & 41 \\
$13 / 2$ & 1.7 & 20 & 73.0 & 49 \\
$13 / 1$ & 4.5 & 8.6 & 78 & 42 \\
$12 / 2$ & 0.8 & 14 & 81 & 41 \\
$11 / 2$ & 10.8 & 12.2 & 92 & 29 \\
$12 / 1$ & 0.2 & 4.2 & 100 & 27 \\
\hline
\end{tabular}

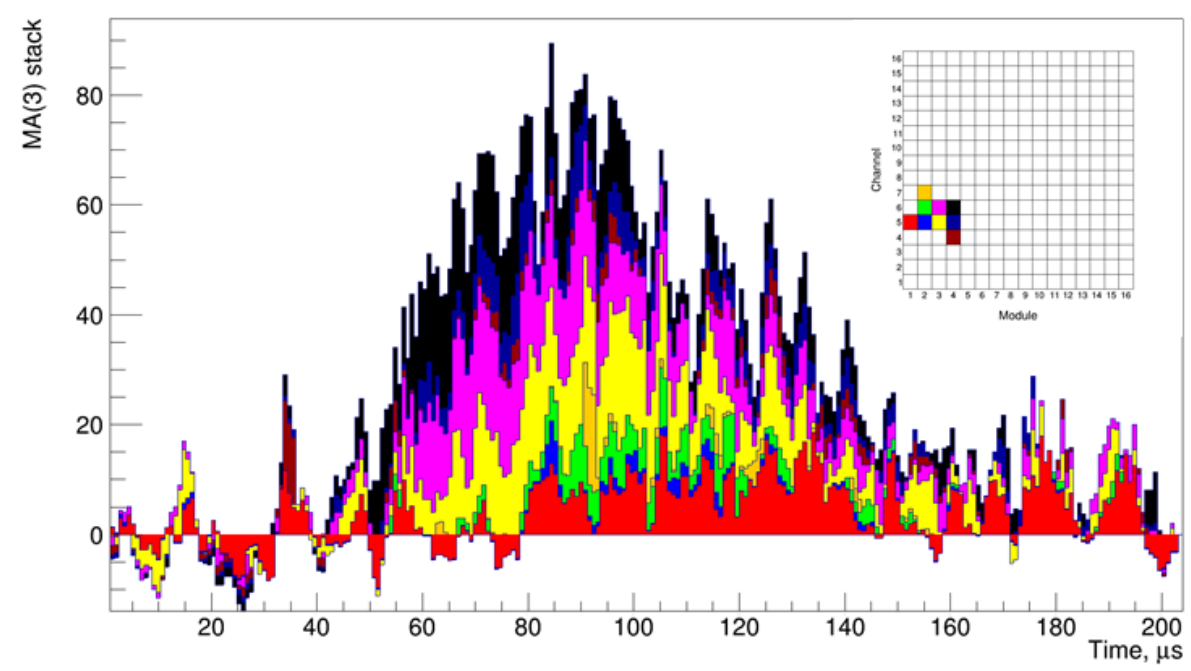

Figure 4: Event 20161031-102518: stack histogram for moving average of ADC counts (with $M=3$ ) for 9 active pixels.

\section{Atmospheric phenomena measurements}

During the first year of operation all four modes of the TUS detector operation were tested. The detector was operating in the TLE mode for two weeks and approximately one month in the METEOR mode. What follows is the examples and brief description of the atmospheric events registered in various modes.

\subsection{ELVES measurements with $0.8 \mu$ s temporal resolution}

The TUS detector measured numerous UV transient flashes in the EAS mode with different temporal dynamics and spatial structure. The most common type of TLEs with a specific geometry 
- bright expanding ring to a maximum radius of $\sim 300 \mathrm{~km}$ - in the ionosphere (altitudes of 80 $90 \mathrm{~km}$ ) are so-called elves ${ }^{3}$ - the result of the ionosphere heating by an expanding electromagnetic wave from a powerful cloud-to-ground lightning. The typical life-time of an elve is $\lesssim 1 \mathrm{~ms}$. Elves represent the most frequent type of TLEs: according to ISUAL (Imager of Sprites and Upper Atmospheric Lightning) global experimental data [35], 50\% of all registered TLEs were determined to be elves.

A number of such events were measured by the TUS detector. The event presented here (see the snapshots of focal plane in Fig.5) was registered on September 18, 2016, above Africa $\left(9^{\circ} .66 \mathrm{~S}, 17^{\circ} .14 \mathrm{~W}\right)$. An arc-like shape of the track made by the brightest PMTs and the speed of development support the hypotheses that this was an elve. Waveforms of several pixels and the geographical location of the event are shown in Fig. 6. The Vaisala GLD360 lightning location network data demonstrate a significant thunderstorm activity in the nearby region. 19 lightning flashes were measured in the area with radius of $500 \mathrm{~km}$ during $\pm 2 \mathrm{~s}$. One of them has a peak current of $135.1 \mathrm{kA}$ which is significantly larger than the threshold of elves production [36].
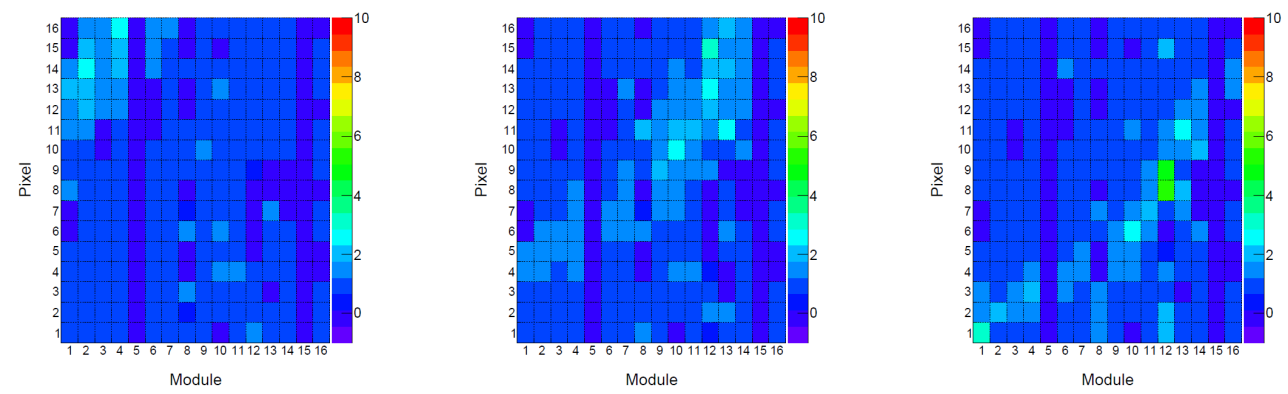

Figure 5: Three snapshots (taken at $t=77 \mu \mathrm{s}, 174 \mu \mathrm{s}$, and $182 \mu \mathrm{s}$ from the beginning of the record) of the focal plane show arc-like shape and movement of the object through the detector's field of view. Colors denote the signal amplitude in arbitrary units scaled to individual PMT gains.

\subsection{Lightning and TLE measurements}

Several hundred of events were measured by the TUS detector in the TLE mode $(0.4 \mathrm{~ms}$ temporal resolution). Most of them represent huge flashes distributed over the entire photo detector. These flashes are lightning discharges under a thundercloud or outside the FOV. In the second case, the measured signal is a diffuse scattered light from the mirror. In some cases the signal has a definite center of luminosity which indicates the position of the events in the FOV. All events have various spatial structure and temporal dynamics. As an example, we present an event that was measured on June 27, 2016, above India, Fig. 7. It occurred in the FOV and produced a huge signal: several pixels in the center of the event are saturated (see also Fig. 8).

A comparison with Vaisala Global Lightning Dataset GLD360 was made for this event. Several lightning strikes were found in this region for the time of the TUS measurements and two of them (negative cloud-to-ground) took place exactly in the FOV. Different events have various

\footnotetext{
3“ELVE" stands for Emission of Light and Very Low Frequency perturbation from an Electromagnetic Pulse (EMP) Sources [34].
} 


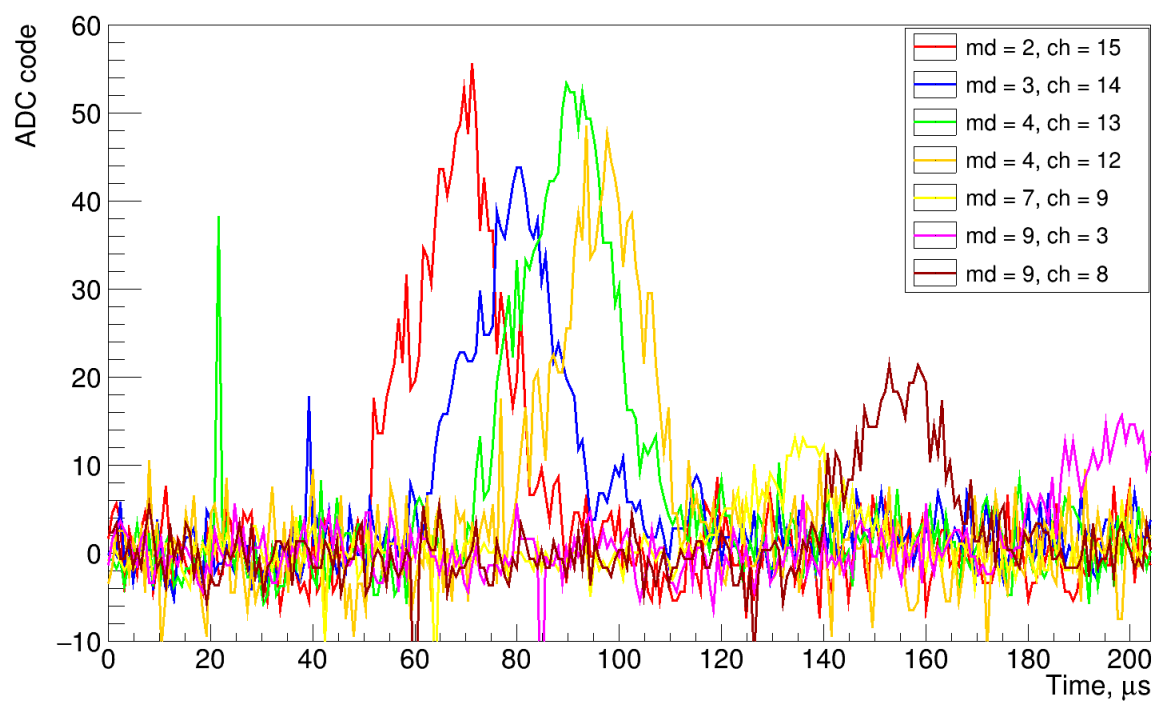

Figure 6: Waveforms of several active pixels of the elve event registered on September 18, 2016, above Africa. The Y-axis is ADC counts for active pixels (individual baselevels are subtracted).

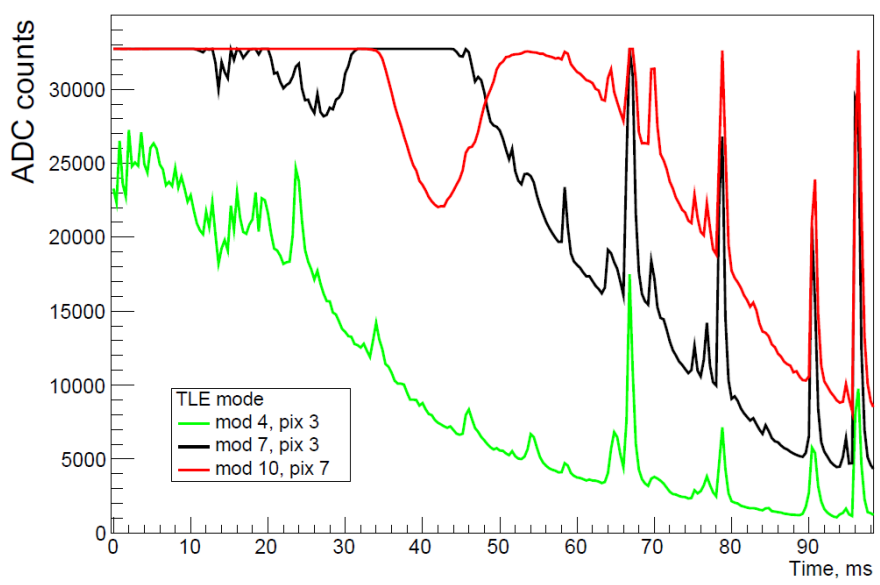

Figure 7: Waveforms of three pixels of the event registered on June 27,2016 , above India $\left(25^{\circ} .3 \mathrm{~S}, 77^{\circ} .8 \mathrm{E}\right)$

temporal structure and most probably occurred in the active thunderstorm regions. The same was observed in previous measurements that were made by Vernov and Tatiana MSU satellites.

\subsection{Meteor measurements}

Unaffected by variable weather conditions, which limit the effectiveness of ground-based meteor observation networks, orbital detector can become an effective facility for meteor study.

The METEOR mode of the TUS operation has $6.6 \mathrm{~ms}$ temporal resolution and it allows to record digital oscillograms of $1.7 \mathrm{~s}$ length. This was implemented for measurements of much 


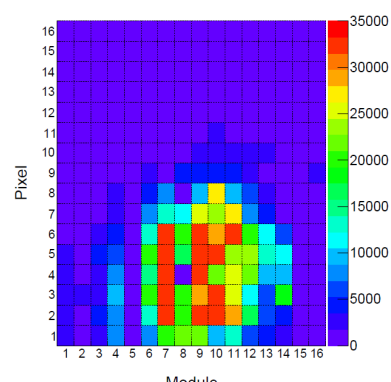

Module

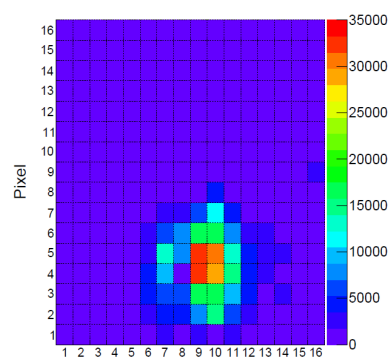

Module

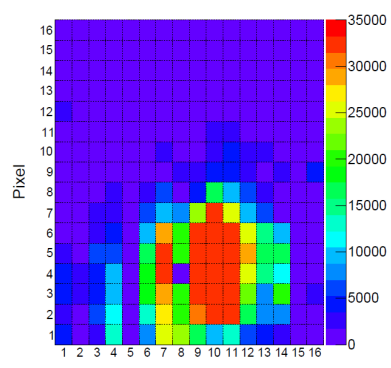

Module

Figure 8: Snapshots (at $t=40 \mathrm{~ms}, 88 \mathrm{~ms}, 96.4 \mathrm{~ms}$ ) of the focal plane for the event from the previous figure. Colors denote ADC counts.

slower than EASs and TLEs processes and it is suitable for registering of meteors, thunderstorms atmospheric airglow and city lights. As it was shown in [37] the standard "solar" meteors with velocities of about $3 \times 10^{6} \mathrm{~cm} / \mathrm{s}$ can be efficiently detected by the fluorescence light produced along the meteor track with the kinetic energy threshold of about $25 \mathrm{~J}$ using the TUS detector with the time sampling near $10 \mathrm{~ms}$.

During the month of operation in this mode a dozen of meteors were measured. One of them is presented in the Fig. 9. The speed of the meteor in assumption of its horizontal movement in the TUS FOV is $\sim 50-60 \mathrm{~km} / \mathrm{s}$. The meteor was registered on January, 3, 2017, at 14:31:08 UTC. This time is close the Quadrantids meteor shower peak in 2017.

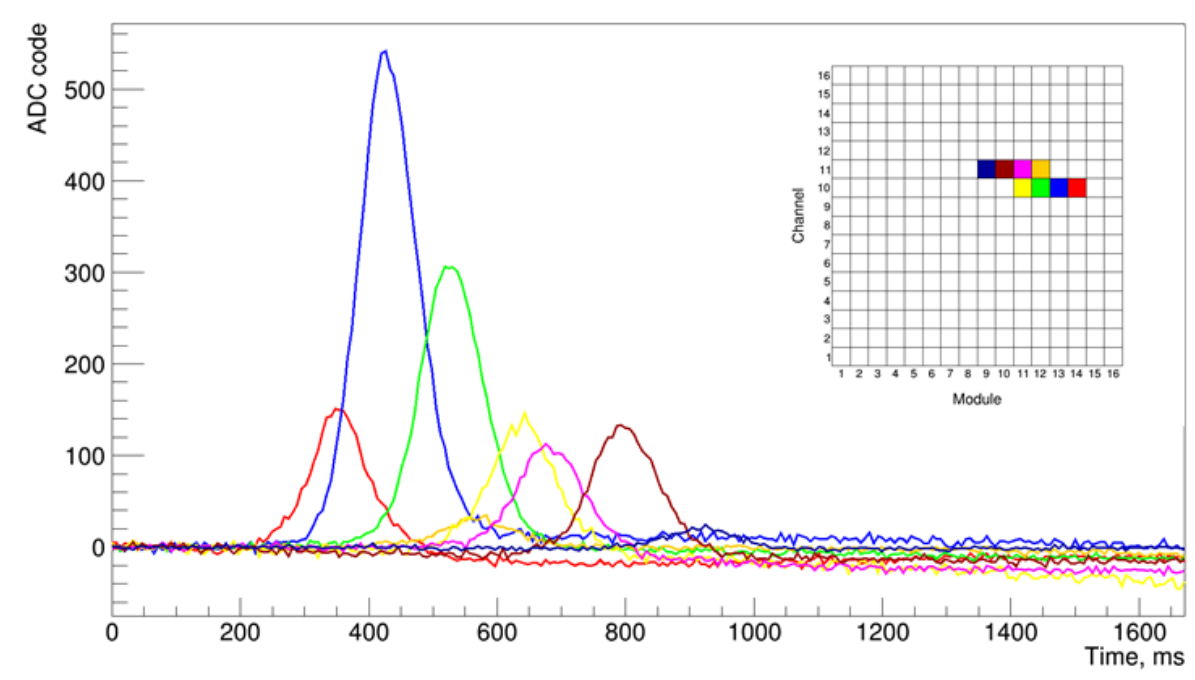

Figure 9: Waveforms of active pixels of the meteor event, measured on January, 3, 2017, at 14:31:08 UTC.

These measurements demonstrate the possibility of usage of EECR fluorescent detector for measurements and study of meteors. This confirms the strategy and estimations made for the next orbital detector for meteor study, discussed in $[38,39]$. It was shown that JEM-EUSO telescope has the capability to observe meteors down to magnitude $M=+7$. Taking advantage of its large 
FOV and high detection rate, JEM-EUSO is able to record a statistically significant flux of meteors, both for sporadic and shower meteors.

\subsection{Thunderstorm measurements with $6.6 \mathrm{~ms}$ temporal resolution}

In the previous space experiments of Moscow State University aimed for the UV atmosphere radiation study a numerous series effects were found. Missions Tatiana-1, Tatiana-2 and Vernov were using a two channel UV and infrared detector based on the same Hamamatsu PMTs and similar electronics as TUS and provide UV measurements with $1 \mathrm{~ms}(0.5 \mathrm{~ms}$ for Vernov satellite) temporal resolution. The events correlated with thunderstorm region usually have multi-peak structure indicating a sequence of UV flashes in the detectors FOV with the inter peak intervals of tens of ms. On the other hand above thunderstorm regions the phenomenon of the series of flashes was observed by Tatiana- 2 experiment: the consecutive transients followed one another with time between the triggers at $T=60 \mathrm{~s}$. In the Vernov experiment, the same approach was used for the search of series and gave similar result, but with a much shorter time interval (due to satellite data transmission capability): $T=4.5 \mathrm{~s}$ between flashes. For more details see [40, 41].

The TUS detector in Meteor mode can measure thunderstorm activity in the time scale which was not covered yet by previous experiments which measure waveforms of 128 or $256 \mathrm{~ms}$ with intervals of $4.5 \mathrm{~s}$. The duration of the meteor mode waveforms is equal $1.7 \mathrm{~s}$. Two examples of measurements are shown in fig. 10. The upper panel shows the measurements above a thunderstorm region which was identified using Vaisala GLD360 data. In the FOV of the detector a series of lightning discharges occurred. Sharp peaks represent the return strokes of powerful lightning flashes. Small and wide peaks represent the other lightning related UV emissions (of the preliminary processes in the thunder clouds, upper atmospheric events etc.). Another event is shown on the bottom panel of the fig. 10. In contrast to the previous one it doesn't have narrow (one sample) peaks. Interesting to note that there were no any lightning measured by Vaisala GLD360 network in the radius of $300 \mathrm{~km}$ from the place of measurements.

\section{Conclusions}

TUS, the first orbital detector of extreme energy cosmic rays, is operating for approximately a year on board the Lomonosov satellite. In a search for an EAS, generated by an EECR, it observes a large number of background events of various origin that take place in the nocturnal atmosphere of the Earth. A multi-level algorithm for the search of EAS-like events was developed and applied to the TUS data set. As a result, a number of preliminary EAS candidates were selected and analysed on the event by event basis according to the spatial-temporal criteria for horizontal and vertical EASs. The most interesting for the further analyses candidates for horizontal EASs, which demonstrate a movement of the image in the FOV were selected. An analysis of their temporal parameters is complicated by the large PSF and different sensitivity of pixels. An influence of possible anthropogenic sources of illumination and weather conditions must also be taken into account. A more detailed analysis of EAS candidate events found in the TUS detector data is in progress, and its results will be reported elsewhere.

Examples of lightning, TLEs and meteors registered by the TUS detector demonstrate the possibility of such an orbital telescope to measure and research the UV transient phenomena of 

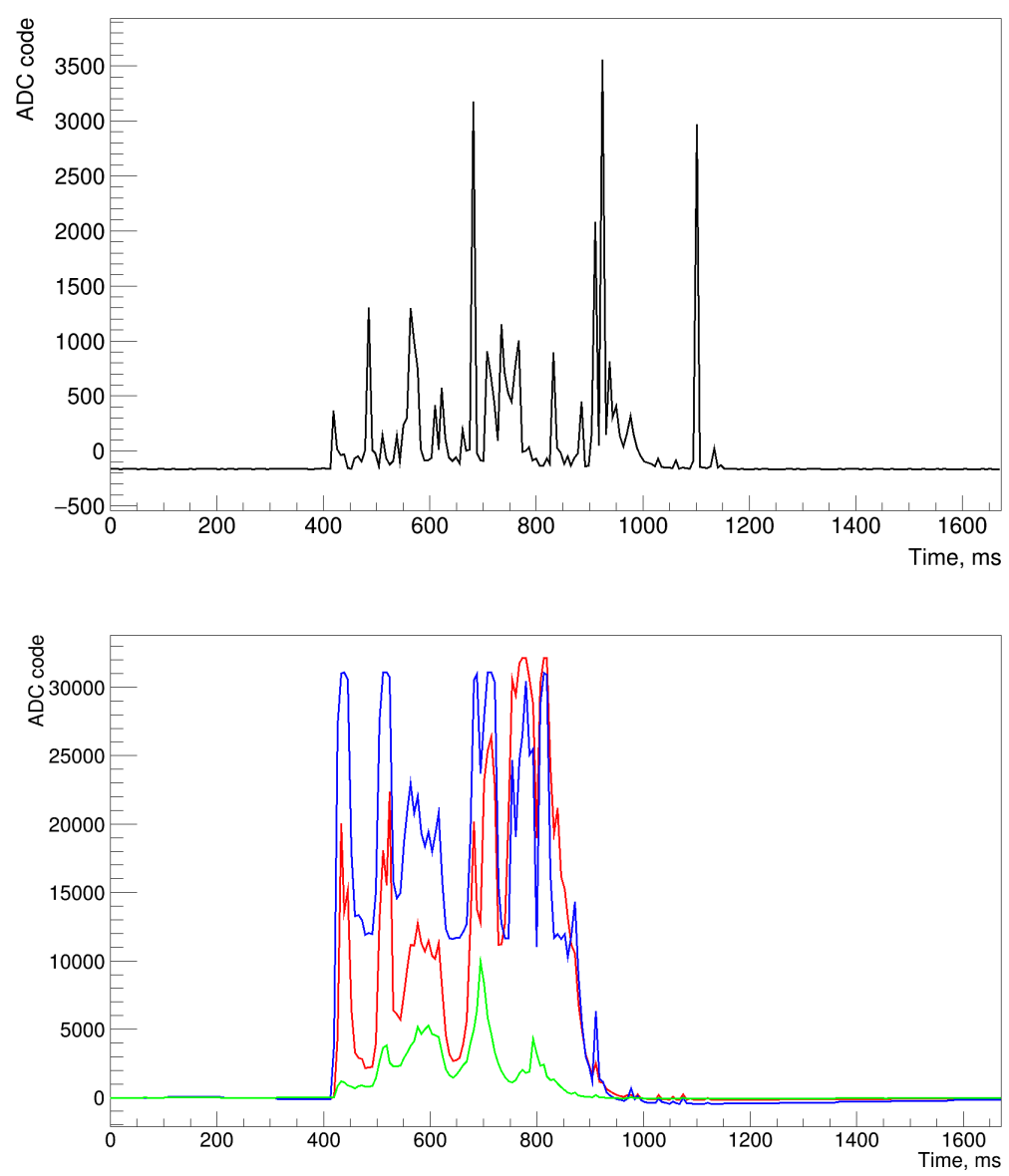

Figure 10: Upper panel: series of UV flashes above thunderstorm. The sharp peaks are supposedly lightning discharges. Bottom panel: far from thunderstorm (based on Vaisala GLD360 network data) UV transient flashes. Different colors correspond to different photodetector channels.

various origin and in different time scales. This feature of the equipment will be used to study the thunderstorm effects in the upper atmosphere.

\section{Acknowledgments}

The authors wish to thank Vaisala Inc. company for providing the data on lightning strikes employed in the present study. The TUS experiment on board the Lomonosov satellite was realized within the Federal Space Program of Russia with funding by the Russian Space Agency and financial support of M.V. Lomonosov Moscow State University through its "Prospects for Development" program ("Perspektivnye Napravleniya Razvitiya"). The data analysis is supported by RFBR grants No. 16-29-13065 and No. 15-02-05498. The Korean work is supported by the National Research Foundation grants (No. 2015R1A2A1A01006870 and No. 2015R1A2A1A15055344). 


\section{References}

[1] B. R. Dawson, M. Fukushima and P. Sokolsky, Past, present and future of UHECR observations, ArXiv e-prints (2017), [1703.07897].

[2] J. Linsley, L. Scarsi and B. Rossi, Extremely Energetic Cosmic-Ray Event, Physical Review Letters 6 (May, 1961) 485-487.

[3] K. Greisen, End to the cosmic-ray spectrum?, Phys. Rev. Lett. 16 (Apr, 1966) 748-750.

[4] G. T. Zatsepin and V. A. Kuz'min, Upper limit of the spectrum of cosmic rays, Soviet Journal of Experimental and Theoretical Physics Letters 4 (Aug., 1966) 78.

[5] V. Berezinsky, Extragalactic cosmic rays and their signatures, Astroparticle Physics 53 (Jan., 2014) 120-129, [1301.0914].

[6] Pierre Auger collaboration, A. Aab et al., Searches for Anisotropies in the Arrival Directions of the Highest Energy Cosmic Rays Detected by the Pierre Auger Observatory, Astrophys. J. 804 (2015) 15, [1411.6111].

[7] TElESCOPE ARrAy collaboration, R. U. Abbasi et al., Indications of Intermediate-Scale Anisotropy of Cosmic Rays with Energy Greater Than 57 EeV in the Northern Sky Measured with the Surface Detector of the Telescope Array Experiment, Astrophys. J. 790 (2014) L21, [1 404.5890 ].

[8] B. A. Khrenov, M. I. Panasyuk, V. V. Alexandrov et al., Space Program KOSMOTEPETL (project KLYPVE and TUS) for the study of extremely high energy cosmic rays, in Observing Ultrahigh Energy Cosmic Rays from Space and Earth (H. Salazar, L. Villasenor and A. Zepeda, eds.), vol. 566 of American Institute of Physics Conference Series, pp. 57-75, May, 2001, DOI.

[9] R. Benson and J. Linsley, Satellite Observation of Cosmic-Ray Air Showers, in Bulletin of the American Astronomical Society, vol. 12 of Bulletin of the American Astronomical Society, p. 818, Sept., 1980.

[10] R. Benson and J. Linsley, Satellite observation of cosmic ray air showers, in 17th International Cosmic Ray Conference, Paris, France, vol. 8, pp. 145-148, 1981.

[11] M. I. Panasyuk, M. Casolino, G. K. Garipov et al., The current status of orbital experiments for UHECR studies, Journal of Physics Conference Series 632 (Aug., 2015) 012097, [1501. 06368 ].

[12] G. K. Garipov, M. Y. Zotov, P. A. Klimov et al., The KLYPVE Ultra High Energy Cosmic Ray Detector on Board the ISS, Bull. Rus. Acad. Sci. Physics 79 (2015) 326-328.

[13] T. Ebisuzaki, G. Medina-Tanco and A. Santangelo, The JEM-EUSO mission, Advances in Space Research 53 (May, 2014) 1499-1505.

[14] B. A. Khrenov, Design and development of space experiments KLYPVE and TUS for study of UHECR, Nuclear Physics B Proceedings Supplements 113 (2002) 115-122.

[15] B. A. Khrenov, V. V. Alexandrov, D. I. Bugrov et al., KLYPVE/TUS space experiments for study of ultrahigh-energy cosmic rays, Physics of Atomic Nuclei 67 (Nov., 2004) 2058-2061.

[16] V. Abrashkin, V. Alexandrov, Y. Arakcheev et al., The TUS space fluorescence detector for study of UHECR and other phenomena of variable fluorescence light in the atmosphere, Advances in Space Research 37 (2006) 1876-1883.

[17] B. A. Khrenov, M. I. Panasyuk, G. K. Garipov et al., Pioneering space based detector for study of cosmic rays beyond GZK Limit, in European Physical Journal Web of Conferences, vol. 53 of European Physical Journal Web of Conferences, p. 09006, June, 2013, DOI. 
[18] J. H. Adams, S. Ahmad, J.-N. Albert et al., Space experiment TUS on board the Lomonosov satellite as pathfinder of JEM-EUSO, Experimental Astronomy 40 (Nov., 2015) 315-326.

[19] P. A. Klimov, M. I. Panasyuk, B. A. Khrenov, G. K. Garipov, N. N. Kalmykov, V. L. Petrov et al., The TUS detector of extreme energy cosmic rays on board the Lomonosov satellite, Space Science Reviews $(8,2017) 1-17$.

[20] A. A. Grinyuk, A. V. Tkachenko, L. G. Tkachev and TUS Collaboration, The TUS orbital detector optical system and trigger simulation, Journal of Physics Conference Series 409 (Feb., 2013) 012105.

[21] A. Grinyuk, V. Grebenyuk, B. Khrenov et al., The orbital TUS detector simulation, Astroparticle Physics 90 (2017) 93-97.

[22] P. Klimov, B. Khrenov, S. Sharakin et al., First results on transient atmospheric events from Tracking Ultraviolet Setup (TUS) on board the Lomonosov satellite, in Proc. of Int. Symposium TEPA-2016 (A. Chilingarian, ed.), pp. 122-127, 2017.

[23] M. Zotov and Lomonosov-UHECR/TLE Collaboration, Early results from TUS, the first orbital detector of extreme energy cosmic rays, ArXiv e-prints (Mar., 2017), [1703. 09484 ].

[24] B. A. Khrenov, P. A. Klimov, M. I. Panasyuk and Lomonosov-UHECR/TLE Collaboration, First results from the TUS orbital detector in the extensive air shower mode, ArXiv e-prints (Apr., 2017), [1704.07704].

[25] S. V. Biktemerova and M. O. Gonchar, Geant4 tool for simulation of arbitrarily defined Fresnel lenses, Physics of Particles and Nuclei Letters 8 (Dec., 2011) 789-793.

[26] L. Tkachev, A. Grinyuk, M. Lavrova and A. Tkachenko, The TUS orbital detector simulation, Proceedings of Science (2015) 610.

[27] C. Berat, S. Bottai, D. De Marco et al., Full simulation of space-based extensive air showers detectors with ESAF, Astroparticle Physics 33 (May, 2010) 221-247, [0 907.5275$].$

[28] N. P. Ilina, N. N. Kalmykov and V. V. Prosin, Cherenkov radiation and parameters of extensive air showers, Sov. J. Nucl. Phys. 55 (1992) 1540-1547.

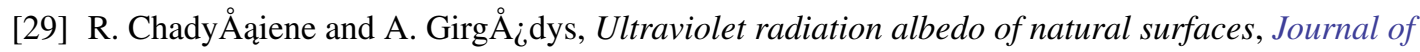
Environmental Engineering and Landscape Management 16 (2008) 83-88, [http://www.tandfonline.com/doi/pdf/10.3846/1648-6897.2008.16.83-88].

[30] S. V. Biktemerova, A. A. Botvinko, N. P. Chirskaya, V. E. Eremeev, G. K. Garipov, V. M. Grebenyuk et al., Search for extreme energy cosmic ray candidates in the TUS orbital experiment data, ArXiv e-prints (June, 2017), [1706.05369].

[31] A. Grinyuk, M. Slunecka, A. Tkachenko, L. Tkachev, P. Klimov and S. Sharakin, The method and results of measurement of the optical parameters of the UHECR detector for the TUS space experiment, Nuclear Instruments and Methods in Physics Research A 763 (Nov., 2014) 604-609.

[32] R. K. Said, U. S. Inan and K. L. Cummins, Long-range lightning geolocation using a VLF radio atmospheric waveform bank, Journal of Geophysical Research (Atmospheres) 115 (Dec., 2010) D23108.

[33] R. K. Said, M. B. Cohen and U. S. Inan, Highly intense lightning over the oceans: Estimated peak currents from global GLD360 observations, Journal of Geophysical Research (Atmospheres) 118 (July, 2013) 6905-6915. 
[34] H. Fukunishi, Y. Takahashi, M. Kubota, K. Sakanoi, U. S. Inan and W. A. Lyons, Elves: Lightning-induced transient luminous events in the lower ionosphere, Geophysical Research Letters 23 (1996) 2157-2160.

[35] A. B. Chen, C.-L. Kuo, Y.-J. Lee, H.-T. Su, R.-R. Hsu, J.-L. Chern et al., Global distributions and occurrence rates of transient luminous events, Journal of Geophysical Research (Space Physics) 113 (Aug., 2008) A08306.

[36] C. P. Barrington-Leigh and U. S. Inan, Elves triggered by positive and negative lightning discharges, Geophysical Research Letters 26 (1999) 683-686.

[37] B. A. Khrenov and V. P. Stulov, Detection of meteors and sub-relativistic dust grains by the fluorescence detectors of ultra high energy cosmic rays, Advances in Space Research 37 (2006) 1868-1875.

[38] J. H. Adams, S. Ahmad, J. N. Albert, D. Allard, L. Anchordoqui, V. Andreev et al., JEM-EUSO: Meteor and nuclearite observations, Experimental Astronomy 40 (Nov, 2015) 253-279.

[39] G. Abdellaoui, S. Abe, A. Acheli, J. Adams, S. Ahmad, A. Ahriche et al., Meteor studies in the framework of the JEM-EUSO program, Planetary and Space Science 143 (2017) 245 - 255.

[40] G. K. Garipov, B. A. Khrenov, P. A. Klimov, V. V. Klimenko, E. A. Mareev, O. Martines et al., Global transients in ultraviolet and red-infrared ranges from data of universitetsky-tatiana-2 satellite, Journal of Geophysical Research: Atmospheres 118 (2013) 370-379.

[41] P. Klimov, G. Garipov, B. Khrenov, V. Morozenko, V. Barinova, V. Bogomolov et al., Vernov satellite data of transient atmospheric events, Journal of Applied Meteorology and Climatology 56 (2017) 2189-2201, [https://doi.org/10.1175/JAMC-D-16-0309.1].

\section{The Lomonosov-UHECR/TLE collaboration}

S.V. Biktemerova ${ }^{b}$, A.A. Botvinko ${ }^{c}$, N.P. Chirskaya ${ }^{a}$, V.E. Eremeev ${ }^{a}$, G.K. Garipov $^{a}$, V.M. Grebenyuk ${ }^{b, d}$, A.A. Grinyuk ${ }^{b}$, S. Jeong ${ }^{f}$, N.N. Kalmykov ${ }^{a}$, M.A. Kaznacheeva ${ }^{a}$, B.A. Khrenov ${ }^{a}$, M. Kim ${ }^{f}$, P.A. Klimov ${ }^{a}$, M.V. Lavrova ${ }^{b}$, J. Lee ${ }^{f}$, O. Martinez $^{g}$, M.I. Panasyuk ${ }^{a}$,

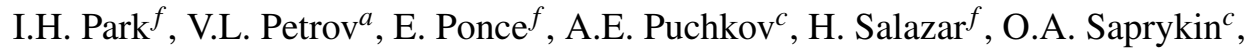
A.N. Senkovsky ${ }^{c}$, S.A. Sharakin ${ }^{a}$, A.V. Shirokov ${ }^{a}$, A.V. Tkachenko ${ }^{b}$, L.G. Tkachev ${ }^{b, d}$, I.V. Yashin ${ }^{a}$, M.Yu. Zotov ${ }^{a}$

${ }^{a}$ M.V. Lomonosov Moscow State University, GSP-1, Leninskie Gory, Moscow, 119991, Russia

${ }^{b}$ Joint Institute for Nuclear Research, Joliot-Curie, 6, Dubna, Moscow region, Russia, 141980

${ }^{c}$ Space Regatta Consortium, ul. Lenina, 4a, 141070 Korolev, Moscow region, Russia ${ }^{d}$ Dubna State University, University str., 19, Bld.1, Dubna, Moscow region, Russia ${ }^{e}$ Department of Physics and ISTS, Sungkyunkwan University, Seobu-ro 2066, Suwon, 440-746 Korea

${ }^{f}$ Benemérita Universidad Autónoma de Puebla, 4 sur 104 Centro Histórico C.P. 72000, Puebla, Mexico 